\title{
Evaluation of Stable Cluster Head Election (SCHE) Routing Protocol for Wireless Sensor Networks
}

\author{
Wan Norsyafizan W.Muhamad, Kaharudin Dimyati, Roslina Mohamad, Rosmalini Abd Kadir
}

\begin{abstract}
The nature of wireless sensor networks necessitates specific design requirement, of which energy efficiency is paramount. One of the important parameter in ensuring a good WSN system is the routing protocol. The energy source in WSN is irreplaceable and their lifetime is limited. Thus, an energy efficient routing protocol is inevitable in WSN. This project present the development of an energy efficient routing protocol which consumes significantly less power compared to existing routing protocol for Wireless Sensor Network. The design was developed based on Low Energy Adaptive Clustering Hierarchy (LEACH) routing protocol, intended to reduce the overall energy consumption. Clustering is an energy efficient and scalable way to organize the WSN. The main objective is to minimize the energy dissipation of each sensor node and reduces the energy dissipation for the whole network. Stable Cluster Head Election (SCHE) analyzes the cluster head selection to find the optimal probability of becoming a cluster head. Simulation of Matlab shows that this design reduces communication energy by as much as 95\% compared to $\mathrm{LEACH}$, due to stable cluster head election.
\end{abstract}

Keywords: Wireless sensor networks, routing protocol, energy efficiency, clustering.

\section{Introduction}

Recent advances in wireless communication and electronics have enabled the development of low cost, low power, multifunctional sensor nodes that are small in size and able to communicate in short distances. This tiny system, which consists of sensing, data processing and communicating components are known as wireless sensor network [1]. WSN is a collection of sensor nodes organized into a cooperative network. It contains hundreds or thousands of sensor nodes. Each node consists of

Wan Norsyafizan W.Muhamad is with Communication Department, Faculty of Electrical Engineering, UiTM., 40450 Shah Alam, Selangor, Malaysia. (email: wnsyafizan@yahoo.com)

Kaharudin Dimyati is with Electrical Department, Faculty of Engineering, UM, 50603, Kuala Lumpur, Malaysia. (email: kahar@um.edu.my)

Roslina Mohamad is with Communication Department, Faculty of Electrical Engineering, UiTM., 40450 Shah Alam, Selangor, Malaysia. (email: roslina780@salam.uitm.edu.my)

Rosmalini Abd Kadir is with Communication Department, Faculty of Electrical Engineering, UiTM., 40450 Shah Alam, Selangor, Malaysia. (email:leeniey79@yahoo.com) processing capability, multiple types of memory, RF transceiver and accommodates various types of sensors and actuators [2]. WSN has limited supply of energy hence an energy conserving routing protocol is important for these networks. Therefore, while conventional networks aim to achieve high quality of service provisions, sensor network focus primarily on power consumption. There are several power-aware routing protocols discussed in the literature $[1,3,4,5]$ and this is also the main target of the work presented in this project. Communication energy conservation can be achieved by limiting the packet size of the data to be sent and by limiting the number of packets that are routed through the network. Computational energy efficiency can be achieved by limiting the number of computational task to be done by a node, in other words making the routing protocol as simple as possible.

\section{Energy Analysis of Routing protocols}

For this project, three existing routing protocols, namely Direct Communication (DC), Minimum Transmission Energy (MTE) and LEACH had been analyzed based on first order radio model [6]. The radio model is illustrated in Figure 1 and the characteristics are summarized in Table 1.

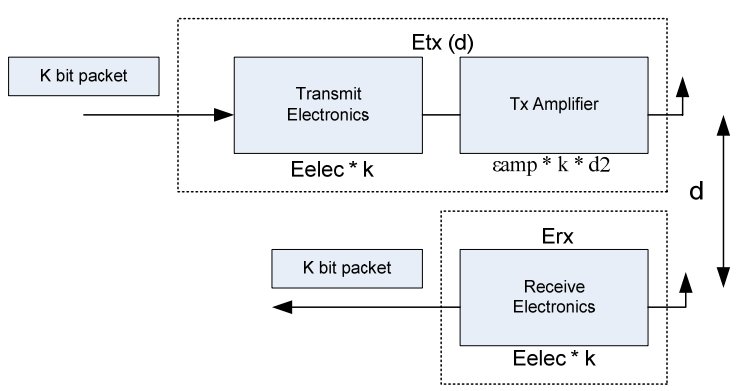

Figure 1: First order radio model

Table 1: radio characteristics

\begin{tabular}{|l|l|l|}
\hline Operation & Energy Dissipated & Definition of variables \\
\hline $\begin{array}{l}\mathrm{E}_{\text {tx-elec }} \\
\mathrm{E}_{\text {rx-elec }} \\
\left(\mathrm{E}_{\mathrm{tx} \text {-elec }}=\mathrm{E}_{\mathrm{rx} \text {-elec }}=\right.\end{array}$ & $50 \mathrm{~nJ} / \mathrm{bit}$ & transmitter electronic \\
$\left.\mathrm{E}_{\text {elec }}\right)$ & & receiver electronic \\
\hline $\mathrm{E}_{\mathrm{amp}}$ & $100 \mathrm{pJ} / \mathrm{bit} / \mathrm{m}^{2}$ & \\
\hline$k$ & & transmit amplifier \\
\hline$d$ & & number of bit message \\
\hline
\end{tabular}


Energy consumption formula for sending a $k$ bit message to a distance $d$ is;

$$
\begin{aligned}
& E_{T x}(k, d)=E_{T x-\text { elec }}(k)+E_{T x-a m p}(k, d) \\
& E_{T x(k, d)}=E_{\text {elec }} * k+\varepsilon_{\text {amp }} * k * d^{2}
\end{aligned}
$$

Energy consumption formula for receiving a $k$ bit message to a distance $d$ is;

$$
\begin{aligned}
& E_{R x}(k)=E_{R x-\text { elec }}(k) \\
& E_{R x}(k)=E_{\text {elec }} * k
\end{aligned}
$$

DC is the simplest topology where all sensor nodes are one hop away from the base station. Consider the linear network shown in Figure 2, where the number of nodes is $n$ and the distance between the nodes is $r$.

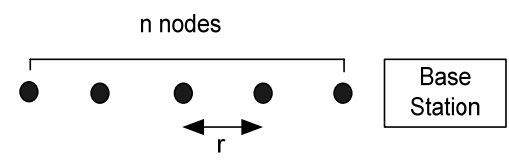

Figure 2: Simple Linear Network

Using the DC approach and equation 1 and equation 2 with consideration energy expanded transmitting a single $k$-bit message from a node located a distance $n r$ from the base station, total energy expanded can be derived as;

$$
\begin{aligned}
& E_{\text {direct }}=E_{T x}(k, d=n * r) \\
& =E_{\text {elec }} * k+\varepsilon_{\text {amp }} * k *(n r)^{2} \\
& =k\left(E_{\text {elec }}+\varepsilon_{\text {amp }} n^{2} r^{2}\right)
\end{aligned}
$$

MTE is a routing protocol for flat topologies where nodes route data destined ultimately for the base station through intermediate nodes. A node acts a router for other nodes. Total energy expanded in the system using MTE routing protocol is;

$$
\begin{aligned}
& E_{M T E}=n * E_{T x}(k, d=r)+(n-1) * E_{r x}(k) \\
& =n\left(E_{\text {elec }} * k+\varepsilon_{\text {amp }} * k * r^{2}\right)+(n-1) * E_{\text {elec }} * k \\
& =k\left((2 n-1) E_{\text {elec }}+\varepsilon_{\text {amp }} n r^{2}\right)
\end{aligned}
$$

LEACH is a self-organizing, adaptive clustering protocol that uses randomization to distribute the energy load evenly among the sensors in the network [7]. Clustering is a very useful approach to reduce energy dissipation in sensor networks [8]. However, there are several problems in LEACH, which make the LEACH routing protocol in the end inefficient. Since the cluster head using more energy than leaf nodes, it is quite important to reselect the cluster head periodically. In order to overcome problem faced by LEACH routing protocol, a new WSN routing protocol was proposed known as SCHE.

SCHE is based on the LEACH architecture which applies clustering. The main objective of the SCHE is to minimize the energy dissipation of each sensor node and reduces the energy dissipation for the whole network. Thus, this new protocol analyze the cluster head selection to find the stable or optimal probability of becoming a cluster head, which will result in suboptimal operation and consumes significantly less power compared to LEACH. An alternative way of reducing energy dissipation which is applied by SCHE is by minimizing the number of transmit/receive message and also by minimizing the distance between the cluster head and the base station.

\subsection{SCHE Algorithm details}

The SCHE was designed with specific criteria such as random network, fixed location of sensor node and base station so that the protocol becomes computational simple as the static route could be used. SCHE is source initiates protocol with time driven reporting, so the sensor node would always have data to send to the base station. SCHE would also apply data aggregation to avoid information overload or access of data, in this aspect SCHE shares the same features as LEACH [7].

SCHE presents an analytic framework for obtaining the stable probability with which a node would become a cluster head in order to minimize the network's energy consumption. The analysis presented for a small network under the assumption of identical expected distance of all cluster heads from the sink. It is important to apply appropriate cluster head election mechanism to reduce the energy consumption for each sensor nodes, which will results in reducing the mean energy consumption for the whole network. This is where SCHE come into picture where it applied this mechanism by obtaining the optimum value of probability for a node of becoming a cluster head which will result in suboptimum operation.

A simple model is assumed which the radio dissipates $E_{\text {elec }} J$ / bit to run the transmitter or receiver circuit and $E_{a m p} J / m^{2}$ for the transmitter amplifier to achieve an acceptable signal to noise ratio. Assuming $r^{2}$ energy loss due to channel transmission, to send a $k$ bit message to a distance of $d$ meters using this radio model, the radio expends

$E_{T x}(k, d)=k E_{\text {elec }}+k \varepsilon_{\text {amp }} d^{2}$

Joules of energy and the radio expands

$$
E_{R x(k, d)}=k E_{\text {elec }}
$$

Joules of energy to receive message. For energy consumption calculation, parameters for SCHE defined [9]. The mean energy consumed by a node in a cycle consists of an energy from cluster head node and also energy from leaf nodes, as given by the following equation 
Proceedings of the International MultiConference of Engineers and Computer Scientists 2010 Vol II, IMECS 2010, March 17 - 19, 2010, Hong Kong

$$
E_{\text {avg }}=p E_{\text {head }}+(1-p) E_{\text {leaf }}
$$

A leaf node needs to turn on the radio during the period of intra cluster communication. Based on the radio model in equation 1 and equation 2 [8], the mean energy consumption of leaf node is given by

$$
\begin{aligned}
& E_{\text {leaf }}=P_{\text {rate }}\left[E_{T x}\left(k_{\text {data }}, r\right)+E_{R x}\left(\frac{T_{\text {int } r a}}{T}-K_{\text {data }}\right)\right] \\
& +\left(1-P_{\text {rate }}\right)\left[E_{R x}\left(\frac{T_{\text {int } r a}}{T}\right)\right.
\end{aligned}
$$

Clusters heads have to turn on their radio during both the intra cluster and the inter cluster periods. Using the radio model in equation 1 and 2, an average energy consumed by a cluster head is given by

$$
\begin{aligned}
& E_{\text {head }}=P_{\text {hrate }}\left[E_{T x}\left(k_{\text {data }}, d\right)+E_{R x}\left(\frac{T_{\text {int } e r}}{T}-k_{\text {data }}\right)\right. \\
& +\left(1-P_{\text {hrate }}\right)\left[E_{R x}\left(\frac{T_{\text {int } e r}}{T}\right)\right]+E_{R x}\left(\frac{T_{\text {int ra }}}{T}\right)
\end{aligned}
$$

Average energy consumed by a node in a cycle can be written as

$$
\begin{aligned}
& E_{\text {avg }}=E_{\text {amp }} k_{\text {data }}\left[\frac{L^{2}(1-p) p_{\text {rate }}}{3 N p}+p\left(1-\left(1-p_{\text {rate }}\right)^{M}\right) d^{2}\right]+ \\
& E_{\text {elec }}\left(k_{\text {intra }}+p k_{\text {inter }}\right)
\end{aligned}
$$

The expected number of nodes in a cluster with every sensor node has the same probability $p$ to become a cluster head, given by

$$
M=\frac{1}{p}
$$

The average energy consumed by a sensor node in the case of small sensor network becomes

$$
\begin{aligned}
& E_{\text {avg }}=E_{\text {amp }} k_{\text {data }} L^{2}\left[\frac{2 p\left(1-\left(1-p_{\text {rate }}\right)^{\frac{1}{p}}\right)+}{3} \frac{(1-p) p_{\text {rate }}}{3 N p}\right] \\
& +E_{\text {elec }}\left[k_{\text {int ra }}+p k_{\text {int er }}\right]
\end{aligned}
$$

Calculation of stable cluster head election probability can be done by taking the derivative of equation 12 with respect to $p$ and equate it to zero. This will give the result of

$$
\begin{aligned}
& 0=E_{\text {elec }} k_{\text {int } e r}-\frac{p_{\text {rate }} E_{\text {amp }} k_{\text {data }} L^{2}+}{3 N p^{2}} \frac{2}{3} E_{\text {amp }} k_{\text {data }} L^{2} \\
& +\frac{2 E_{\text {amp }} k_{\text {data }} L^{2}\left(1-p_{\text {rate }}\right)^{\frac{1}{p}}}{3}\left[\frac{\ln \left(1-p_{\text {rate }}\right)-1}{p}\right]
\end{aligned}
$$

Subsequently, the stable probability of a sensor node to become a cluster head is derived from equation 13 as a function of $P_{\text {rate }}$. Assume that all the nodes have data to send in each cycle $\left(P_{\text {rate }}=1\right)$, the stable cluster head election probability $(p)$ becomes

$$
p=\sqrt{\frac{E_{\text {amp }} K_{\text {data }} L^{2}}{N\left(3 E_{\text {elec }} K_{\text {inter }}+2 E_{\text {amp }} L^{2} k_{\text {data }}\right)}}
$$

This is the optimal value of $p$.

\section{Results and Discussions}

Simulations were performed to evaluate the energy dissipation achieved by LEACH and SCHE routing protocol. It can be divided into three cases, for the first case, consider a hundred sensor nodes and the distance between each cluster head to the sink set as $10 \mathrm{~m}$ for the scenario where each node has a 2000 bit data packet to send to the cluster head, subsequently, the cluster head will forward the message to the base station.

For the second case, simulation of the transmission of data from the cluster head to the base station was done for two different distances, which are $10 \mathrm{~m}$ and $5 \mathrm{~m}$. Finally simulations were conducted for both protocols, consider the scenario where each cluster head has a 2000 and 1000bit data packet to send to the base station.

\subsection{Comparison between LEACH and SCHE for energy dissipation.}

Simulation was performed for both LEACH and SCHE routing protocol to compare the energy dissipation. LEACH routing protocol using random probability to evaluate energy dissipation whereas SCHE routing protocol applied stable cluster head probability to evaluate the energy dissipation. Result for this simulation shows in Figure 3.

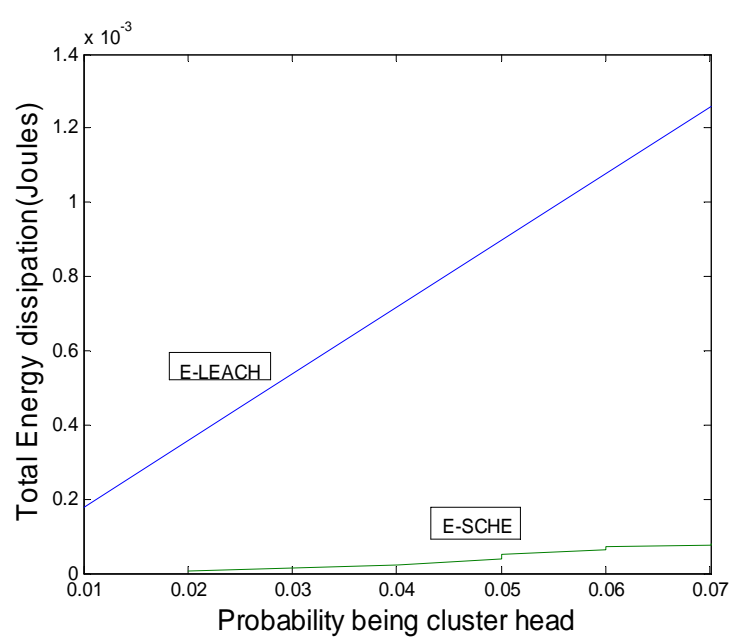

Figure 3: Energy dissipation for LEACH and SCHE 
Figure 3 shows the relationship between the total energy dissipated for both LEACH and SCHE routing protocol versus the probability with which the nodes becoming a cluster head for a network with a hundred sensor nodes and the distance between each cluster head to the sink was assumed to be $10 \mathrm{~m}$. From the graph, it could be concluded that SCHE routing protocol consumes less energy compared to LEACH routing protocol, with the 95\% reduction. This is due to the stable cluster head election probability. LEACH routing protocol applies the random cluster head election, which will result in much energy consumption. This shows that effective selection of cluster head could reduce the usage of consumption power.

\subsection{Evaluating the energy dissipation for LEACH and SCHE with different transmission distance.}

In this section, consider two scenarios where the distance between each cluster head and the sink are $10 \mathrm{~m}$ and $5 \mathrm{~m}$ respectively. The relationship between the energy dissipation for both protocols with difference $d$ and the probability of becoming a cluster head are shown in Figure 4.

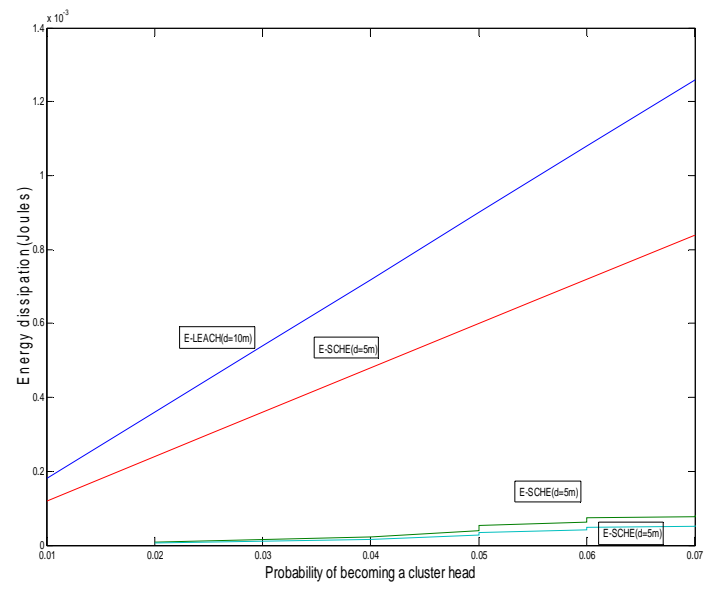

Figure 4: Energy dissipation for LEACH and SCHE with different $d$.

From figure 4, it could be concluded that the energy dissipation for both protocols show an average percentage of reduction of energy dissipation when reducing the distance between the cluster head and the sink. But still SCHE routing protocol results in less energy dissipation compared to LEACH routing protocol for both distance. It can be concluded that minimizing the transmission distance from $10 \mathrm{~m}$ to $5 \mathrm{~m}$ accordingly results in reduction of the energy dissipation

\subsection{Evaluating the energy dissipation for LEACH and SCHE for different number of transmit message.}

Simulation was conducted using hundred random sensor nodes and had each sensor node to send a 2000 bit data packet to the cluster head and subsequently the cluster head would forward the message to the sink. Consider a small network with hundred sensor nodes and the distance between each cluster head to the sink is $10 \mathrm{~m}$. Then simulation followed with the bit message changed to 1000 bit. Results obtained from both simulations are illustrated in the Figure 5.

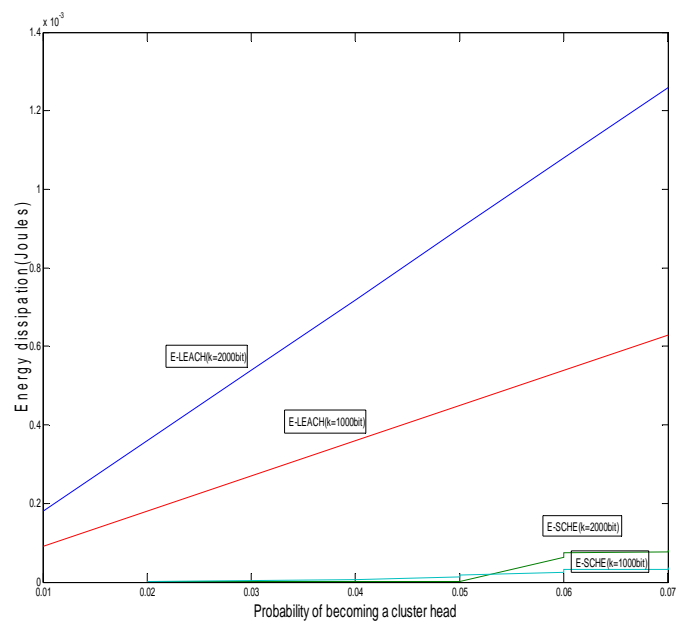

Figure 5: Energy dissipation for LEACH and SCHE with different number of bit message.

Figure 5 shows that, as predicted [6], when the number of transmit or receive messages reduced from 2000 bit to 1000 bit messages, there is a reduction in the energy dissipation for LEACH and SCHE routing protocols. It could be seen that LEACH achieves $50 \%$ reduction in the energy dissipation when the number of transmit/receive message reduced. The same result is obtained for SCHE routing protocol, which achieves an average of $60 \%$ reduction in the energy dissipation when the number of bit message reduced.

\section{Conclusions}

In WSN power efficiency is an important performance metrics that must be considered when designing the routing protocol. Objective of this project is to design an energy aware routing protocol based on LEACH. Results show that LEACH is an energy efficient routing protocol when compared to conventional routing protocol, which are DC and MTE. However, there are several problems in LEACH, which make the LEACH routing protocol also in the end inefficient. SCHE was proposed to overcome the problems faced by the LEACH routing protocol. The SCHE fills the void that exists in the current literature for an energy efficient routing protocol. It is based on the LEACH architecture which applies clustering. The main objective of the SCHE is to minimize the energy dissipation of each sensor node and reduces the energy dissipation for the whole network. This new protocol analyze the cluster head selection to find the stable or optimal probability of becoming a cluster head, which will result in suboptimal operation and consumes significantly less power compared to LEACH. 
Proceedings of the International MultiConference of Engineers and Computer Scientists 2010 Vol II, IMECS 2010, March 17 - 19, 2010, Hong Kong

Simulation results show that SCHE reduces communication energy by as much as $95 \%$ compared to LEACH. This is due to the stable cluster head election, which results in optimal probability of becoming a cluster head. It could be concluded that an effective selection of a cluster head could reduce the usage of consumption power. Simulation also shows by minimizing the transmission distance of SCHE and LEACH would result in an average of $30 \%$ reduction for the energy dissipation. Also, when reducing the number of transmit or receive message, LEACH shows $50 \%$ of reduction and SCHE an average of $60 \%$ reduction in energy dissipation.

\section{References}

[1] I.F Akyildiz, W.Su, Y.Sankarasubramaniam, E.Cayirci, "A Survey on Wireless Sensor Networks," IEEE Communications Magazines, vol 40, No 8, pp 102-114, August 2002.

[2] M.Ilays, Imam Mahgoub, Handbook of Sensor Networks: Compact Wireless and Wired Sensing Systems, CRC Press, Boca Raton, USA, 2004.

[3] Q.Jiang, D.Manivann, "Routing Protocols for sensor networks," Proceedings of the $1^{\text {st }}$ IEEE Consumer Communications and Networking Conference, Las Vegas, USA, January 2004, pp93-98.

[4] H.S Kim, K.J Han,”A Power Efficient Routing Protocol based on Balanced Tree in the WSN," Proceedings of the $1^{\text {st }}$ International Conference on Distributed Framework for Multimedia Applications, February 2005, pp 138-143.

[5] Selvadurai Selvakennedy, “An Energy Efficient Clustering Algorithm for Multihop data Gathering in Wireless Sensor Networks," Journal of Computers, vol 1, no 1, April 2006.

[6] W.R Heinzelman, A.Chandrakasan, H.Balakrishnan, "Energy Efficient Communication Protocol for Wireless Sensor Networks," Proceedings of the $33^{\text {rd }}$ Hawaii International Conference on System Science, January 2000.

[7] Jamil Ibriq, Iman Mahgoub, "Cluster Based Routing in Wireless sensor Networks:Issues and Challenges," Proceedings of the 2004 Symposium on Performance Evaluation of Computer Telecommuincations System, July 2004.

[8] Kemal Akkaya, Mohamed Younis, "A Survey on routing Protocol for Wireless Sensor Networks," Elsevier Ad Hoc Journal, vol 3, no 3, pp325-349.

[9] Wan Norsyafizan W.Muhamad, Evaluation of Stable Cluster Head Election (SCHE) Routing Protocol for WSN, M. Eng, Faculty of Electrical Engineering, University of Malaya, Kuala Lumpur, August 2008. 